\title{
miR-378 inhibits cell growth and enhances apoptosis in human myelodysplastic syndromes
}

\author{
XINGYI KUANG ${ }^{1}$, CHUNMEI WEI $^{1}$, TAO ZHANG $^{1}$, ZESONG YANG $^{1}$, JIANXIANG CHI $^{2}$ and LI WANG $^{1}$ \\ ${ }^{1}$ Department of Hematology, The First Affiliated Hospital of Chongqing Medical University, Chongqing 400016, \\ P.R. China; ${ }^{2}$ The Center for the Study of Haematological Malignancies, 2032 Nicosia, Cyprus
}

Received April 16, 2016; Accepted July 13, 2016

DOI: $10.3892 /$ ijo.2016.3689

\begin{abstract}
R-378 has been proven to inhibit cell growth, migration and invasion in different types of cancers. In this study, we found that miR-378 was commonly downregulated in the bone marrow cells obtained from myelodysplastic syndrome (MDS) patients. We further investigated the role of miR-378 in the proliferation and apoptosis of SKM-1 cells, an acute myeloid leukemia cell line established in the leukemic phase during the progression of MDS to AML (MDS/AML). Results indicated that overexpression of miR-378 in SKM-1 cells interfered with proliferation via inducing apoptosis and G0/G1-phase cell cycle arrest, and suppressive effect of miR-378 on MDS/AML cells may be mediated partly through Bcl-w and CDC40. Moreover, apoptosis induced by miR-378 correlated with increased expression of Bax and activation of caspase-3, -8 and -9 . Taken together, our data support a critical role for miR-378 in the pathogenesis of MDS and provide a novel therapeutic target in this complex disease.
\end{abstract}

\section{Introduction}

MDS is one of the most common blood cancers among the elderly, and $\sim 33 \%$ patients will develop into acute myeloid leukemia (AML). In fact, MDS are a class of heterogeneous clonal disorders that derive from primitive $\mathrm{CD}_{3}{ }^{+}$stem cells and present with diverse clinical symptoms and outcomes (1). Regarding therapy, the only way to cure this disease is haematopoietic stem cell transplantation (HSCT). Unfortunately,

Correspondence to: Dr Li Wang, Department of Hematology, The First Affiliated Hospital of Chongqing Medical University, 1 Youyi Road, Yuzhong, Chongqing 400016, P.R. China

E-mail: liwangls@yahoo.com

Abbreviations: MDS, myelodysplastic syndrome; AML, acute myeloid leukemia; HSCT, haematopoietic stem cell transplantation; miRNAs, microRNAs; BM-MNCs, bone marrow mononuclear cells; Bcl-2, B cell lymphoma 2

Key words: microRNA-378, myelodysplastic syndrome, acute myeloid leukemia, Bcl-w, CDC40
HSCT is only applicable for a small number of MDS patients owing to a variety of factors such as advanced age, various comorbidities, and donor availability (2). Moreover, prognoses are still bleak in MDS patients. The median survival ranges from 68.4 months in the low-risk group to 4.8 months in the high-risk group, and the prognosis is even worse in secondary AML (3). Current knowledge of the molecular mechanisms of MDS is still limited, even though haploinsufficiency, gene mutations, epigenetic deregulation, microenvironmental changes have been proven to be critical contributors in the pathophysiology of this disease (4). Therefore, it is important to clarify the molecular pathogenesis of MDS and to explore novel therapy for clinical use.

microRNAs (miRNAs) are short single-stranded RNAs that inhibit gene expression at the post-transcriptional level (5), and over half of miRNA genes are located in cancer-related genomic regions (6). In recent years, increasing studies have shown that miRNAs are implicated in normal hematopoiesis (7), and dysregulation of miRNA has been found in hematological malignancies including MDS (8-11). More importantly, deregulation of miRNA emerges as displaying a key role in the pathogenesis of MDS (12-15). For example, expression of miR-143 and miR-145 is diminished in MDS with del(5q) and knockdown of these two miRNAs in mice results in abnormal erythropoiesis and megakaryopoiesis, which are important features of 5q- syndrome (one subtype of MDS with isolate 5q-) (15). miR-22 is upregulated in MDS patients, and its downregulation in leukemic cells inhibits cell proliferation via targeting TET2 (14).

With the use of miRNA microarray or real-time PCR, aberrant expression of miR-378 has been detected in a number of cancers. Expression of miR-378 was downregulated in the specimens of colorectal cancer, gastric cancer, and cutaneous squamous cell carcinoma (16-18), whereas it is overexpressed in nasopharyngeal carcinoma, breast cancer, and ovarian cancer (19-21). More importantly, miR-378 has been reported to be implicated in the tumorigenesis of various solid cancers $(16,17,19,22)$. In non-small cell lung cancer, miR-378 exerts oncogenic activity to promote cell migration, angiogenesis and tumor growth via SUFU-related and VEGF/MMP pathways (22). In contrast, miR-378 performs as a tumor suppressor in gastric cancer and colorectal cancer by targeting VEGF and vimentin, respectively $(16,17)$. However, to date, the majority of functional studies of miR-378 focus on solid cancers, 
only a few studies have investigated the physiological role of miR-378 in hematologic malignancies. In leukemia cells, miR-378 is upregulated during the differentiation induced by 4-hydroxynonenal (23), and miR-378 is related to prognosis of AML patients (24). A recent miRNA profiling study showed that miR-378 was the most potently downregulated miRNA in MDS patients (25), providing evidence that miR-378 may be of significance for the pathogenesis of MDS. However, the functional role of miR-378 has not been reported in MDS.

In this study, we detected the expression of miR-378 in bone marrow mononuclear cells (BM-MNCs) of $20 \mathrm{MDS}$ patients and 13 healthy controls. The functional effect of miR-378 was further explored through cell growth, apoptosis and cell cycle analyses in MDS-derived cell line SKM-1 (26). Moreover, xenograft experiments in NOD/SCID mice were operated to investigate the potential role of miR-378 in MDS tumorigenesis in vivo. Finally, we went onto detail the mechanisms that mediated the suppressive effect of miR-378 on MDS/AML cells.

\section{Materials and methods}

Patients and specimens. For miR-378 expression analysis, bone marrow samples were obtained from 20 newly diagnosed MDS patients at The First Affiliated Hospital of Chongqing Medical University. Mononuclear cells were separated from bone marrow by Ficoll gradient centrifugation. The diagnosis of MDS were established according to 2008 WHO criteria. 5 patients had refractory anemia, 3 had refractory anemia with ringed sideroblasts, 2 had refractory anemia with multilineage dysplasia, 5 patients with refractory anemia with excess blasts, 1 with 5 q- syndrome, 1 patient had secondary AML, and 3 with MDS unclassified. Among these MDS patients, male to female ratio was $12: 8$ and average age was 58.1 years (range 39-80). Non-malignant bone marrow samples were collected from 13 randomly selected healthy people, 6 men and 7 women, with a mean age of 60.2 years (range 40-83). This study was approved by the institutional review board of The First Affiliated Hospital of Chongqing Medical University and all patients provided informed consent.

Cell culture. SKM-1 cell line was kindly provided by Professor Jianfeng Zhou from Tongji Medical College of Hua Zhong University of Science and Technology. The cell line was cultured in RPMI-1640 medium (Gibco, Carlsbad, CA, USA) supplemented with $10 \%$ fetal bovine serum (Gibco) and $1 \%$ penicillin/streptomycin (Beyotime, Shanghai, China) at $37^{\circ} \mathrm{C} / 5 \% \mathrm{CO}_{2}$.

Lentiviral vector construction and lentivirus transfection. To obtain SKM-1 cells with miR-378 overexpression, the premiRNA of miR-378 was cloned into the GV209 lentiviral vector (Genechem, Shanghai, China). The primers for cloning pre-mir-378 were: forward sequence 5'-CGGGTACCGGTAC AGCTGGAGAAAGAGGCTG-3' and reverse sequence 5'-CGGAATTCAAAAAATTCTCTTGCGGCACCAC-3'. The production, purification, and titration of lentivirus were performed as described by Tiscornia and colleagues (27). Recombinant lentivirus carrying miR-378 was named LV-miR-378, and the lentivirus expressing empty vector was called LV-control. At 30-50\% confluence, typically $24 \mathrm{~h}$ after plating, SKM-1 cells were transfected with LV-miR-378 and $\mathrm{LV}$-control using $5 \mu \mathrm{g} / \mathrm{ml}$ polybrene (Genechem). After 6 days, the transfection efficiency was evaluated using fluorescence microscopy and flow cytometry, and the cells stably overexpressing miR-378 were confirmed by real-time PCR.

Cell counting kit (CCK-8) proliferation assay. Cell proliferation was evaluated using a CCK-8 assay after 5-day lentivirus infection. SKM-1 ells were seeded into 96-well plates at a density of $5 \times 10^{3}$ cells/well with 3 replicate wells of each condition. For the CCK- 8 assay, $10 \mu \mathrm{l}$ of CCK-8 solution (Beyotime) was added to each well and incubated at $37^{\circ} \mathrm{C}$ for $2 \mathrm{~h}$. Absorbance values at $450 \mathrm{~nm}$ were measured using the SpectraMax M2 Multi-Mode Microplate Readers (Molecular Devices, Silicon Valley, CA, USA) daily for four consecutive days.

Apoptosis detected by Hoechst 33258 staining. Cells were fixed and washed twice in PBS. Then, the fixed cells were stained with $500 \mu$ l Hoechest 33258 solution (Beyotime) in dark for $5 \mathrm{~min}$. Then the cells were observed under a fluorescence microscope.

Annexin V-PE/7-AAD staining for apoptosis analysis. Approximately $10^{6}$ cells were harvested and incubated for $15 \mathrm{~min}$ in staining solution containing $500 \mu \mathrm{l}$ of binding buffer, $1 \mu \mathrm{l}$ of Annexin V-PE and $5 \mu \mathrm{l}$ of 7-AAD (KeyGen Biotech, Shanghai, China). After that, apoptosis rate of cells were detected by flow cytometry using CellQuest software (BD Biosciences, San Jose, CA, USA).

Cell cycle analysis. Cells were collected and fixed in $75 \%$ ethanol at $4^{\circ} \mathrm{C}$ overnight, then incubated in staining cocktail containing $50 \mu \mathrm{g} / \mathrm{ml}$ propidium iodide (PI) and $50 \mu \mathrm{g} / \mathrm{ml}$ RNase for $30 \mathrm{~min}$ at $37^{\circ} \mathrm{C}$. DNA content of samples were analyzed with the use of FACSVantage flow cytometer (BD Biosciences) and Multicycle software.

Quantitative reverse transcriptase-polymerase chain reaction ( $q R T-P C R)$. Quantitative RT-PCR was carried out to validate the expression level of miR-378. Total RNA was extracted from cells using RNAiso Plus (Takara, Dalian, China), digested with DNase I and reversely transcribed with the use of Prime Script ${ }^{\mathrm{TM}}$ RT reagent kit (Takara). Each reverse transcription reaction was performed in $10 \mu \mathrm{l}$ reaction volume containing $0.1 \mu \mathrm{g}$ of total RNAs, $2 \mu \mathrm{l}$ of $5 \mathrm{X}$ PrimeScript buffer, 250 fmol special stem-loop RT primer (Novland, Shanghai, China), and $0.5 \mu \mathrm{l}$ of PrimeScript RT Enzyme Mix I. Reverse transcription conditions were as follows: $16^{\circ} \mathrm{C}$ for $30 \mathrm{~min}, 42^{\circ} \mathrm{C}$ for $45 \mathrm{~min}$, and $85^{\circ} \mathrm{C}$ for $10 \mathrm{~min}$. The reverse transcription products were amplified using a SYBR Premix Ex Taq ${ }^{\mathrm{TM}}$ II kit (Takara) on the Bio-Rad CFX96 system at $95^{\circ} \mathrm{C}$ for $30 \mathrm{sec}$, followed by 40 cycles at $95^{\circ} \mathrm{C}$ for $5 \mathrm{sec}$, and at $60^{\circ} \mathrm{C}$ for $30 \mathrm{sec}$. U6 was amplified as a reference for normalization. For mRNA quantification, $\beta$-actin was selected as the internal control. Total RNA was reverse transcribed with the use of Prime Script ${ }^{\mathrm{TM}}$ RT reagent kit (Takara) following the manufacturer's instructions. Quantitative PCR analysis were performed in real-time using SYBR Premix Ex $\mathrm{Taq}^{\mathrm{TM}}$ II kit 
(Takara) on the Bio-Rad CFX96 system. Real-time PCR specific primers are shown as follows: Bcl-w F: 5'-GCCTT G TTCCTTCATCATCC-3', Bcl-w R: 5'-TACCTAAGCCTCCAC CCATC-3'; miR-378 F: 5'-CTATTAAACGACTGGACTT GGAG-3', miR-378 R: 5'-TATGCTTGTTCTCGTCTCTG TGTC-3'; $\beta$-actin F: 5'-CCACGAAACTACCTTCAACTAA-3', $\beta$-actin R: 5'-GTGATCTCCTTCTGCATCCTGT-3'; U6 F: 5'-ATTGGAACGATACAGA-GAAGATT-3', U6 R: 5'-GGA ACGCTTCACGAATTTG-3'.

Western blot analysis. Protein lysate was extracted from cells using RIPA lysis buffer supplemented with $1 \mu \mathrm{M}$ PMSF (Beyotime), and protein concentration was measured with BCA Protein Assay kit (Beyotime). Western blotting was performed as previously described (28). The following primary antibodies were used in this study: rabbit anti-human cleaved-caspase-3, cleaved-caspase-8, cleaved-caspase-9, Bcl-w and Bax (immunoway, Newark, DE, USA); rabbit polyclonal antibody against human CDC40 (Abcam, Cambridge, UK); horseradish peroxidase-conjugated goat anti-rabbit IgG (Beyotime) was used as second antibody in this study. GAPDH served as a loading control. All results were visualized with the use of BeyoECL Plus (Beyotime).

Dual-luciferase reporter assay. For dual-luciferase reporter assay, the wild-type and mutated 3'-UTR of Bcl-w mRNA was cloned into the dual luciferase reporter vectors. HEK 293T cells were seeded into 24 -well plates and cotransfected with the luciferase-repoter vectors and miR-378/negative control vectors. After 48-h transfection, luciferase activity was detected using the Dual-Luciferase Reporter Assay system (Promega) according to the instructions.

Tumor growth experiments in vivo. Female 5- to 6-weekold NOD/SCID mice were obtained from Beijing HEK Bioscience. The mice were subcutaneously injected with $1 \times 10^{7}$ SKM-1 cells (suspended in $200 \mu 1$ of RPMI-1640 medium) that infected with LV-miR-378 or LV-control. After 4 weeks, mice were sacrificed, and their tumors were carefully excised. Tomor voulmes were meaursed and calculated using the following formula: volume $\left(\mathrm{mm}^{3}\right)=\mathrm{LxW}^{2} / 2$ ( $\mathrm{L}$ represents the largest diameter; $\mathrm{W}$ is the smallest diameter of tumor). All animal experiments were approved by the Ethics Committee of Chongqing Medical University.

Histological analysis. The xenograft tumors were fixed in $4 \%$ paraformaldehyde solution, embedded in paraffin and cut into $3-\mu$ m-thick sections. Then the paraffin sections were stained with hematoxylin and eosin (H\&E). DNA fragmentation in nucleus, which is a typical features of apoptosis, can be detected by the terminal dUTP nick-end labeling (TUNEL) assay. We employed TUNEL assay to evaluate the cell apoptosis in situ using a TUNEL apoptosis assay kit (Beyotime) according to the manufacturer's instructions. Cell counting was conducted on four random fileds of each section, and cells with dark brown nuclei were identified as apoptotic cells. Final data were expressed as the percentages of apoptotic cells.

Statistical analysis. All statistical analyses were performed with SPSS software 20.0. Results were presented as mean \pm

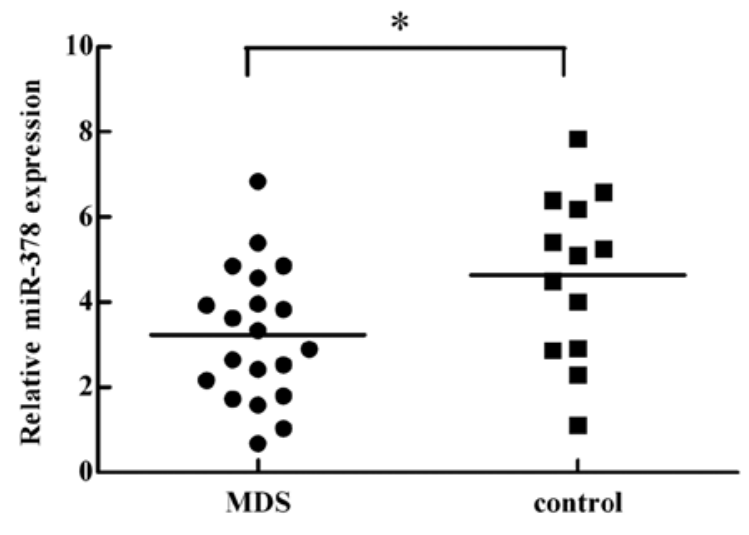

Figure 1. The relative expression of miR-378 in BM-MNCs of MDS patients $(n=20)$ and healthy controls $(n=13) .{ }^{*} P<0.05$ compared with healthy controls.

standard deviation (SD). Two-tailed Student's t-test was carried out to determine significance when only two groups were compared. Variations between groups were analyzed with one-way ANOVA, followed by Tukey multiple comparison test. $\mathrm{P}<0.05$ was considered statistically significant.

\section{Results}

Expression level of $m i R-378$ in MDS patients. BM-MNCs are widely used samples in the miRNA profiling studies of MDS. They are unsorted cells containing not only stem cells but also maturing white cells, which may fully reflect aberrant expression of miRNAs and maximize total RNA yield from the precious patient samples. With the utilization of qRT-PCR, we demonstrated that the expression of miR-378 is obviously decreased in BM-MNCs from MDS patients in contrast with healthy controls (Fig. 1).

Upregulation of miR-378 by lentivirus in SKM-1 cells. To clarify the role of miR-378 in the human MDS, SKM-1 cells were transfected with LV-miR-378 for upregulating the expression of miR-378. After infection of LV-miR-378, SKM-1 cells were observed under fluorescence microscope to generally evaluate the infection efficiency (Fig. 2A and B). By using flow cytometry, we found the percentage of green fluorescent protein (GFP)-positive cells was $>70 \%$ after transfection with LV-miR-378 (Fig. 2C), suggesting that the lentivirus successfully transduced into SKM-1 cells. Next, expression of miR-378 was detected using qRT-PCR. Lentivirus expressing miR-378 significantly increased the miR-378 level in SKM-1 cells (Fig. 2D).

Overexpression of miR-378 inhibited SKM-1 cell growth in vitro. To investigate the effect of miR-378 on cell proliferation, we conducted CCK- 8 assay. After 3 days, the OD value was significantly decreased in SKM-1 cells transfected with LV-miR-378 compared with cells transfected with LV-control and untransfected SKM-1 cells (Fig. 3). The results proved that overexpression of miR-378 inhibited cell growth ability in SKM-1 cells.

Effects of miR-378 on apoptosis and cell cycle of SKM-1 cells. The effect of miR-378 on SKM-1 cell apoptosis was assessed 
A

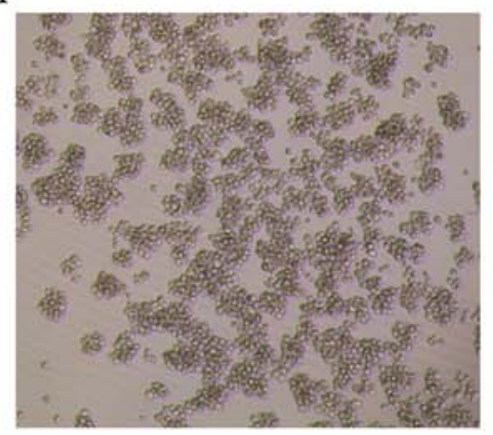

B

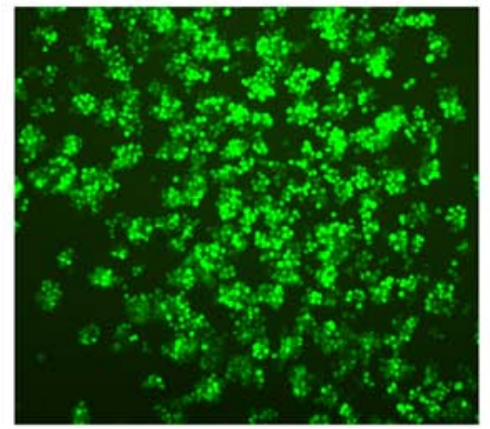

$\mathrm{C}$

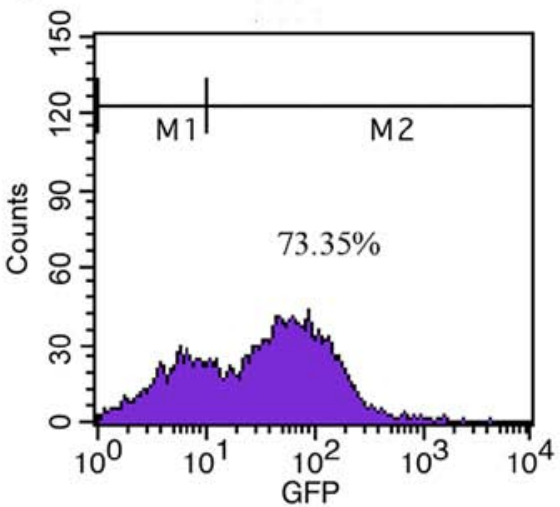

D

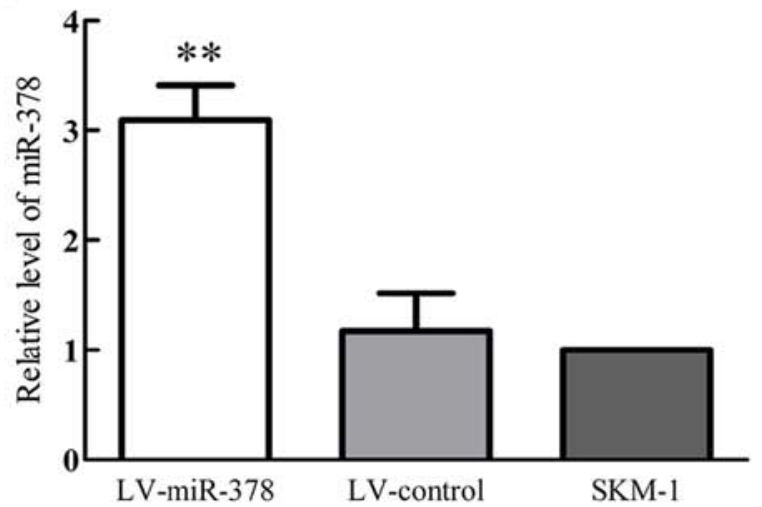

Figure 2. LV-miR-378 was successfully introduced into SKM-1 cells. (A) The SKM-1 cells infected with LV-miR-378 were observed under the bright field (x100). (B) The SKM-1 cells infected with LV-miR-378 were observed under the green fluorescent field of fluorescence microscope (x100). (C) The infection efficiency was examined by flow cytometry. (D) Expression of miR-378 was significantly increased in LV-miR-378 transfected group. ${ }^{* *} \mathrm{P}<0.01$.

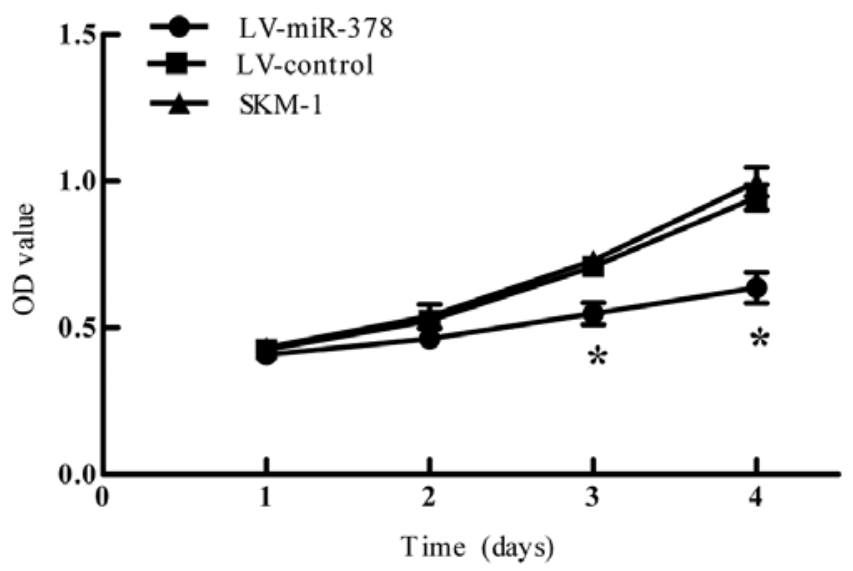

Figure 3. Effect of miR-378 on SKM-1 cell growth in vitro. After two days of LV-miR-378 infection, the growth of SKM-1 cells was obviously inhibited. ${ }^{*} \mathrm{P}<0.05$.

using Annexin V/7-AAD staining assay. It showed that overexpression of miR-378 induced apoptosis in SKM-1 cells, and the percentage of apoptotic cells in miR-378 overexpressing group was significantly higher than that of other two groups (Fig. 4A and D). We also observed apoptotic state in each group by Hoechst 33258 staining. Fluorescence microscope imaging displayed that more cells in miR-378 overexpressing group showed apoptotic morphological characteristics (Fig. 4B). In addition, cell cycle distribution was evaluated using flow cytometry. Results showed that miR-378 significantly increased the proportion of cells in G0/G1 phase and decreased the percentage of cells in S phase (Fig. 4C and E).

Overexpression of miR-378 inhibits tumor growth and promotes apoptosis in vivo. To observe the effect of miR-378 on tumor growth in vivo, we conducted NOD/SCID mice models in which mice were transplanted with LV-miR-378-treated SKM-1 cells, LV-control-treated SKM-1 cells and normal SKM-1 cells. As a result, infection of LV-miR-378 resulted in a significant inhibition on tumor growth. In addition, overexpression of miR-378 obviously reduced the volume and weight of SKM-1 cell-derived tumors (Fig. 5A and B). H\&E staining of xenograft tumors showed disorderly and irregular tumor cell arrangement and increased nucleo-cytoplasmic ratio, which is consistent with the pathological characteristics of malignancy (Fig. 5C). Moreover, TUNEL assay revealed the proportion of apoptotic cells was higher in the miR-378 overexpressing group $(37.08 \pm 7.65 \%)$ than in the LV-control-treated group $(17.49 \pm 3.13 \%, P=0.001)$ and the untreated SKM-1 cell group $(16.93 \pm 2.95 \%, P=0.001$, Fig. 5D).

miR-378 induces apoptosis in SKM-1 cells by activating both intrinsic and extrinsic apoptosis pathway. To further investigate the specific mechanisms by which miR-378 induces apoptosis in MDS cells, we employ western blot assay to detect the expression of caspase- 3, $-8,-9$ and Bax. As shown in Fig. 6, upregulation of miR-378 obviously increased the expression of 


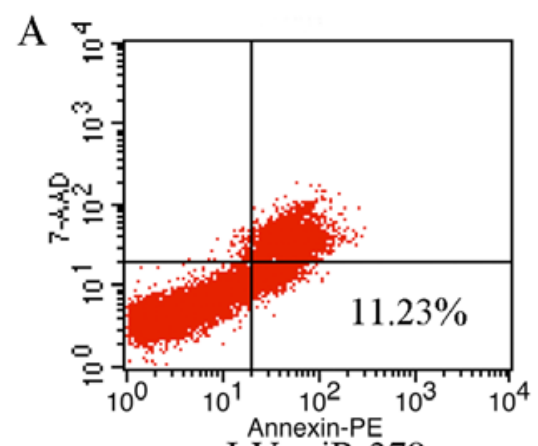

LV-miR-378

\section{B}

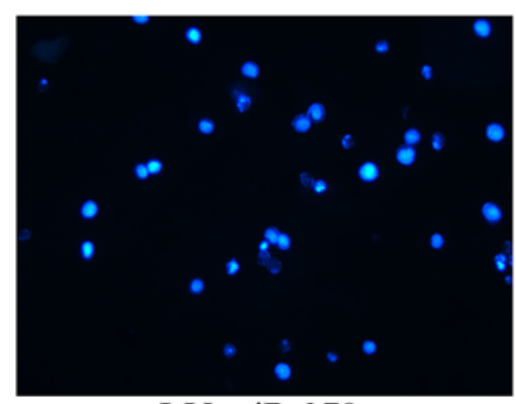

LV-miR-378

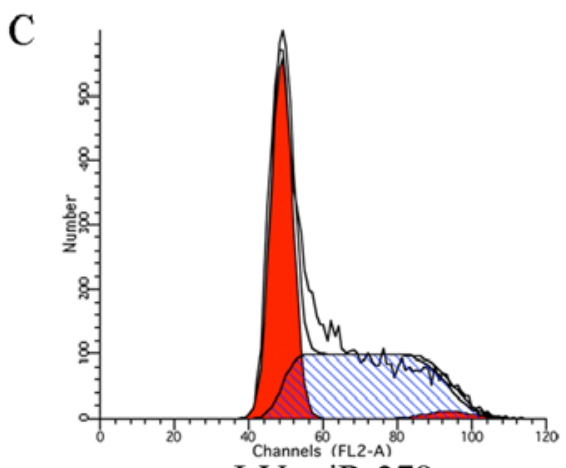

LV-miR-378

D

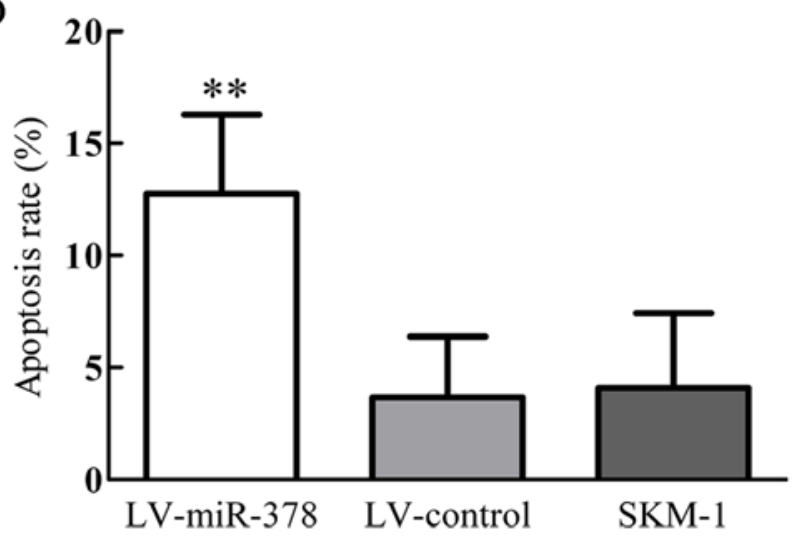

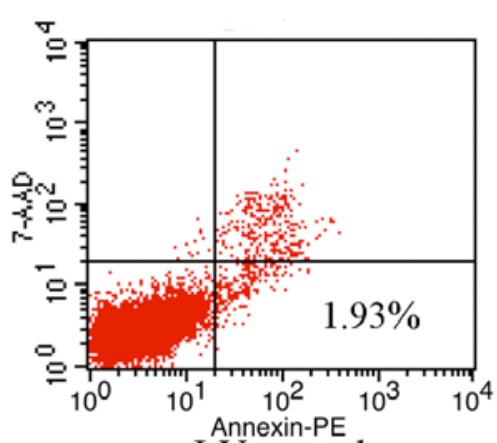

LV-control

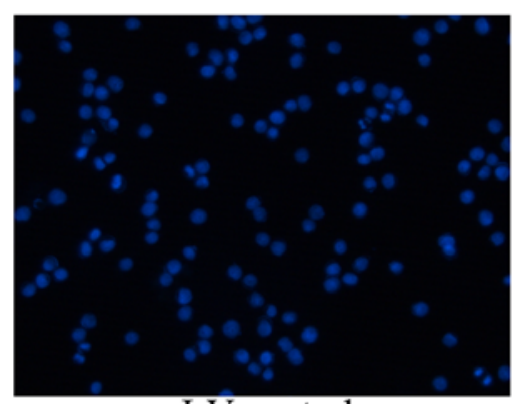

LV-control

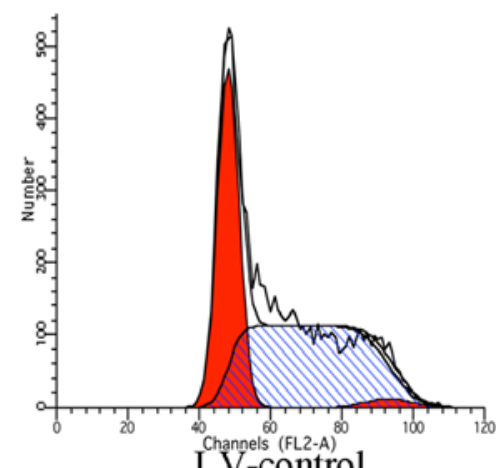

E

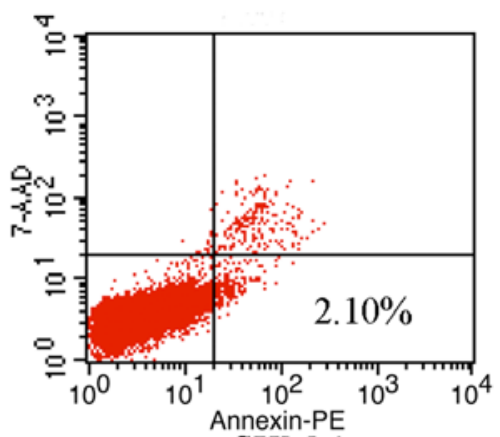

SKM-1

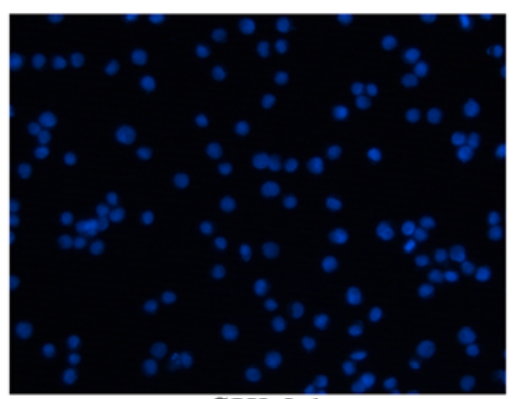

SKM-1

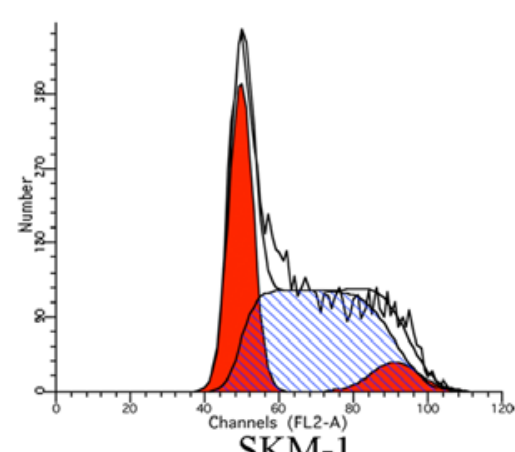

SKM-1

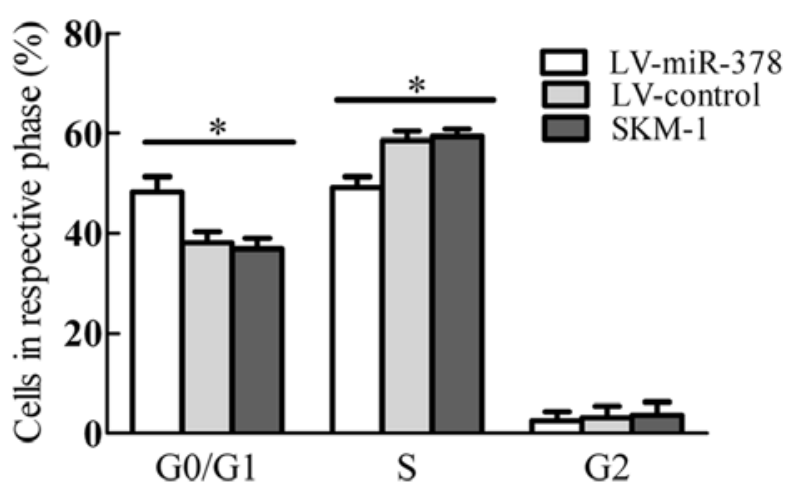

Figure 4. miR-378 suppresses SKM-1 cell growth by inducing cell apoptosis and G1-phase cell cycle arrest. (A) Cell apoptosis was assessed by Annexin V/7-AAD staining after infection with LV-miR-378 and LV-control. (B) Apoptotic cells were also detected by nuclear staining with Hoechst 33258. (C) Representative images depicting cell cycle distribution of SKM-1 cells overexpressing miR-378, SKM-1 cells transfected with LV-control and normal SKM-1 cells, respectively. (D) Apoptosis rate of SKM-1 cells transfected with LV-miR-378 significantly increased, when compared with LV-control transfected SKM-1 cells and normal SKM-1 cells. ${ }^{* *} \mathrm{P}<0.01$ compared with other two groups. (E) Proportion of cells in various phases of the cell cycle. The proportion of LV-miR-378 transfected cells increased in G0/G1 phase, but it decreased in S phase. ${ }^{*} \mathrm{P}<0.05$ compared with the other two groups.

cleaved-caspase-3, -8 and -9 . Also, overexpression of miR-378 led to upregulated expression of proapoptotic protein Bax.
Taken together, these results confirmed that overexpression of miR-378 activated caspase-dependent apoptosis in MDS cells. 
A

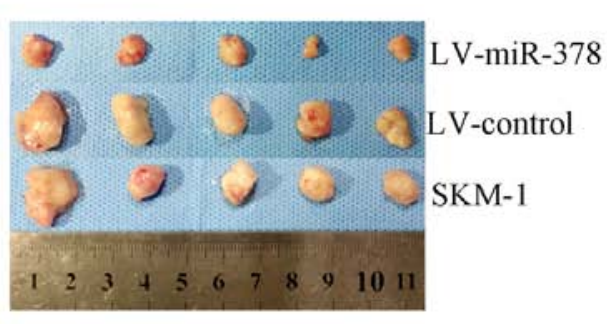

C

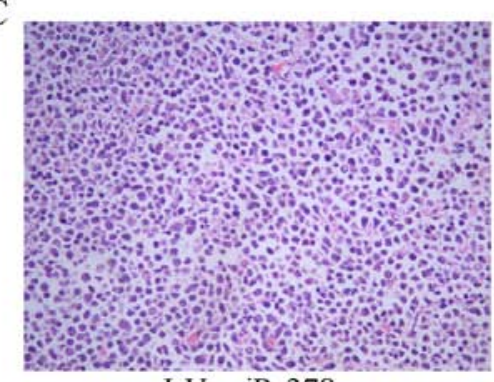

LV-miR-378

D

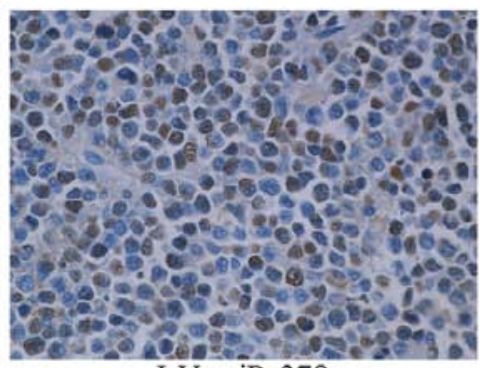

B
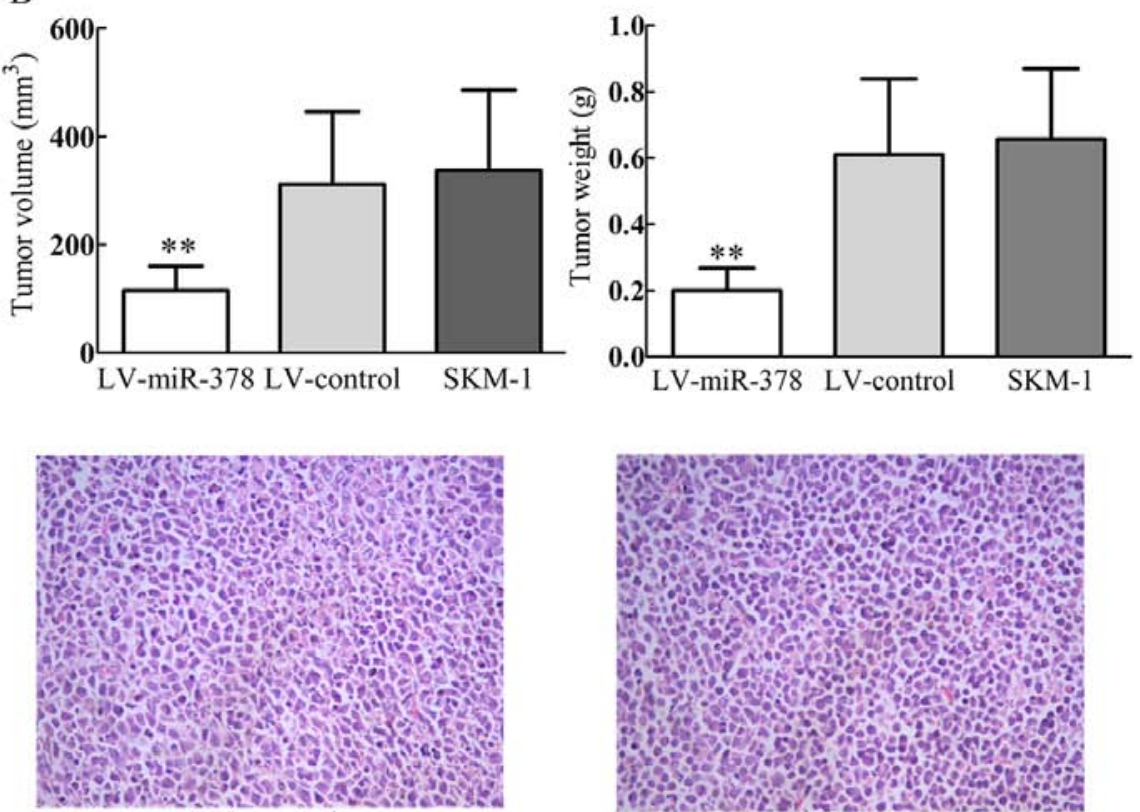

LV-control

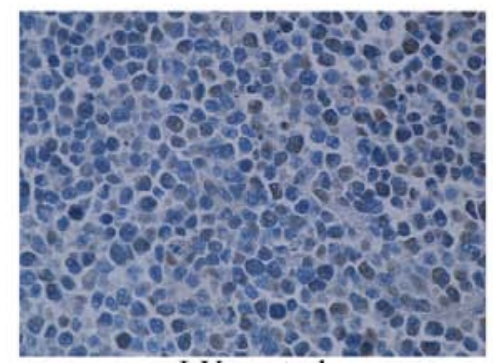

LV-control

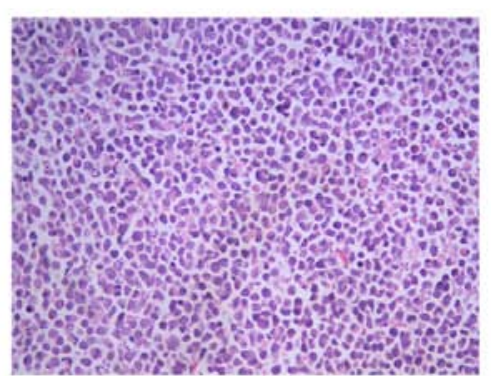

SKM-1

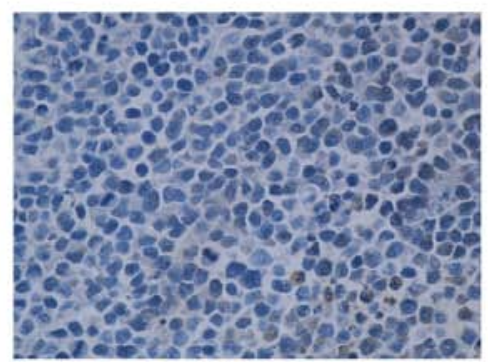

SKM-1

Figure 5. Effect of miR-378 on SKM-1 cell-derived tumor growth in vivo. (A) Images of tumors obtained from NOD/SICD mice in respective groups. (B) Volume and weight of xenograft tumors were both decreased in miR-378 overexpressing group compared with two control groups. ** $\mathrm{P}<0.01$ versus other two groups. (C) H\&E staining of xenograft tumors in respective groups (x200). (D) Representative images of TUNEL staining (x400). Cells with deep brown nuclei were identified as TUNEL-positive cells.

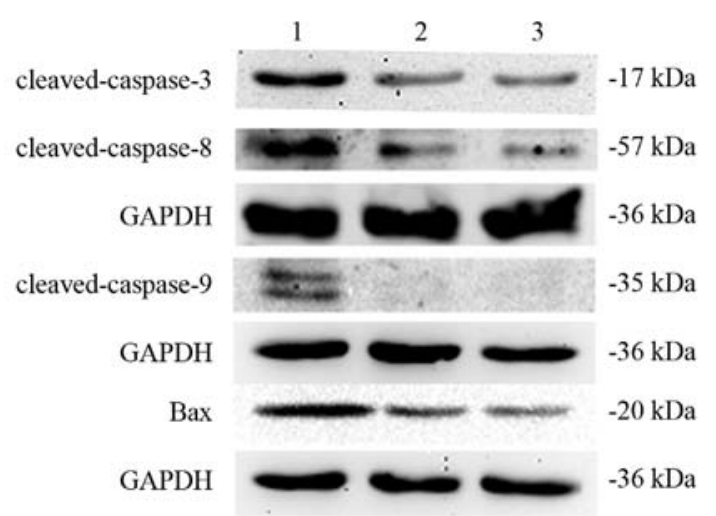

Figure 6. Overexpression of miR-378 upregulated the expression of cleavedcaspase- $3,-8,-9$ and Bax protein. The expression of cleaved-caspase- $3,-8,-9$ and Bax were evaluated by western blot analysis. GAPDH was used as a loading control. Lane 1, LV-miR-378 group; lane 2, LV-control group; lane 3, SKM-1 group.

Expression of target genes are suppressed by miR-378. To explore the apoptosis-related target genes of miR-378 in
SKM-1 cells, we performed bioinformatics algorithms Targetscan (http://www.targetscan.org/) and PicTar (http:// pictar.mdc-berlin.de/). Both bioinformatic analyses indicated that anti-apoptotic protein gene Bcl-w is a putative target of miR-378. Then we conducted dual-luciferase reporter assay to determine whether Bcl-w is a direct target of miR-378. As shown in Fig. 7B, overexpression of miR-378 resulted in a remarkable diminution of luciferase activity in $293 \mathrm{~T}$ cells transfected with Bcl-w wild-type 3'UTR vector. By contrast, there was no obvious change of luciferase activity in SKM-1 cells cotransfected with miR-378 vectors and Bcl-w mutanttype 3'UTR vectors. We further conducted qRT-PCR and western blotting to determine whether overexpression of miR-378 could result in a downregulation of Bcl-w expression. As a result, overexpression of miR-378 in SKM-1 cells resulted in a marked decrease of Bcl-w protein expression level, whereas the expression of Bcl-w mRNA was not affected obviously (Fig. 7C). We also examined the expression of CDC40, which is a proven target of miR-378 (29), by using a western blot assay. It showed that miR-378 significantly diminished the expression of CDC40 in SKM-1 cells (Fig. 7D). 
A

$\begin{array}{ll}\text { hsa-miR-378 } & \text { 3' GGAAGACUGAGGUUCAGGUCA 5' } \\ \text { Bcl-w WT } & \text { 5' GCCUCAGGUGUUGAGUCCAGA 3' } \\ \text { Bcl-w MUT } & \text { 5' GCCUCAGGUGUUGCUGAACUA 3' }\end{array}$

C

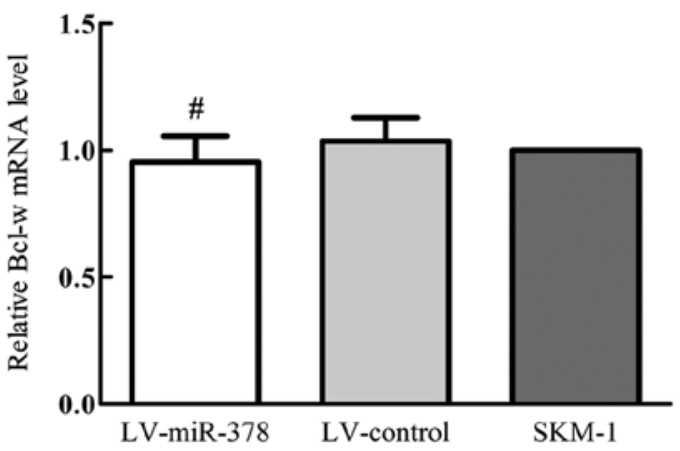

D

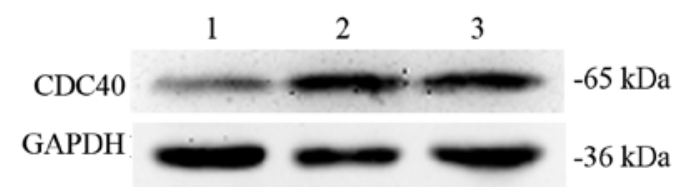

B
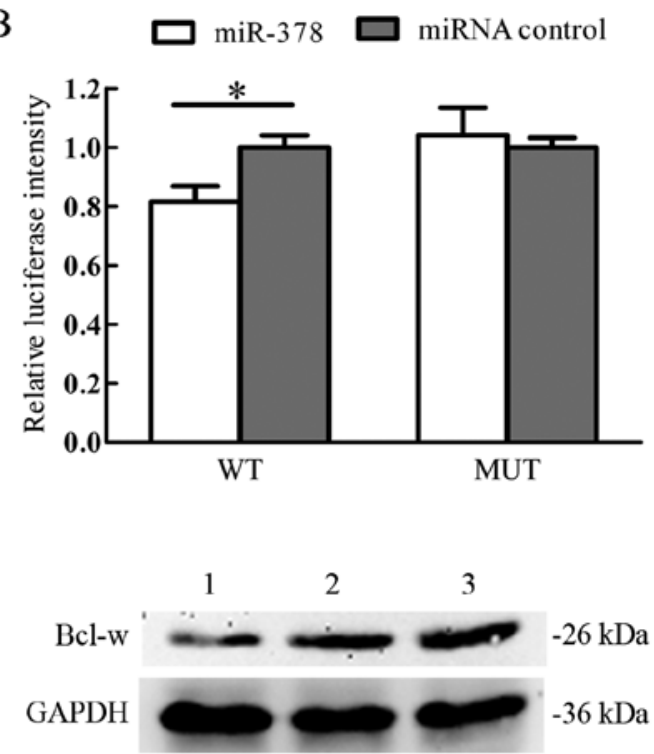

Figure 7. Bcl-w is a direct target of miR-378, and the expression of CDC40 (which is a validated target of miR-378) is also downregulated by miR-378 in SKM-1 cells. (A) The wild-type (WT) and mutated (MUT) 3'UTR of Bcl-w, with seed region and base substitutions (bold). (B) Enforced miR-378 expression inhibited WT but not MUT Bcl-w 3'UTR reporter luciferase activity. The histograms show the normalized luciferase intensity. ${ }^{*} \mathrm{P}<0.05$, versus miRNA control group. (C) The expression levels of Bcl-w mRNA and protein were detected by qRT-PCR and western blot assays, respectively. ${ }^{\# P}>0.05$, versus the other two control groups (D) Expression level of CDC40 protein was examined using western blot assay. Lane 1, LV-miR-378 group; lane 2, LV-control group; lane 3, SKM-1 group.

\section{Discussion}

miR-378 has been proven to participate in the initiation and development of various cancers $(16,19,29,30)$. However, the role of miR-378 in cancer cells seems controversial because it plays a tumor-promting role in non-small cell lung cancer and nasopharyngeal carcinoma $(19,30)$, but exerts tumorsuppressive effect on gastric cancer and colorectal cancer $(16,29)$. Recently, a study identified markedly downregulated expression of miR-378 in BM-MNCs from MDS patients (25), suggesting that miR-378 may participate in the molecular pathogenesis of MDS. However, the knowledge of its pathogenetic role in MDS is still lacking.

In this study, we identified a lower expression level of miR-378 in MDS patients compared with healthy controls. SKM-1 is a malignant cell line established in the leukemic phase during MDS progress towards AML (26), and it is an eligible model to investigate the mechanisms underlying the pathogenesis and transformation of MDS (31). In functional studies, we observed that overexpression of miR-378 in SKM-1 cells attenuated cell proliferation in vitro by promoting cell apoptosis and blocking cell cycle progression. These results indicated that miR-378 played a tumor-suppressive role in the MDS/AML cell line, and it may contribute to the transformation of MDS to AML. However, the effect of miR-378 is heterogeneous because it exerts a totally reverse role in different types of cancers, which may due to miR-378 targeting different genes in these cancers and further regulates diverse signaling pathways. In agreement with our results, Wang et al (29) found that miR-378 inhibited colorectal cancer cell growth via inducing cell cycle arrest and apoptosis. They further proved that CDC40, a critical cellcycle regulator promoting G1/S-phase transition (32), was a direct target of miR-378 and responsible for cell cycle arrest in colorectal cancer cells. We also found the enhanced expression of miR-378 increased CDC40 protein expression, presenting a potential mechanism by which miR-378 caused G1-phase cell cycle arrest in SKM-1 cells.

Our in vitro study showed that overexpression of miR-378 could inhibit proliferation and promote apoptosis of SKM-1 cells, suggesting that miR-378 played an inhibitory role in the pathogenesis and progression of MDS. However, the initiation and development of cancer is not only related to the characteristics of the cancer cells, but also closely related to the complex interaction between the cancer and the host or the microenvironment in vivo (33). The immunodeficient mouse models, especially those of SCID mice and NOD/SCID mice, have been used to study the growth and tumorigenesis of hematological malignant tumors in vivo $(34,35)$. In order to determine whether miR-378 overexpression could affect the growth and apoptosis of MDS cells in vivo, we conducted 
NOD/SCID mouse models in which mice were subcutaneously injected with LV-miR-378-treated SKM-1 cells, LV-controltreated SKM-1 cells and untreated SKM-1 cells. The xenograft experiments in NOD/SCID mice revealed that miR-378 could suppress tumor growth in vivo, strengthening the pathogenetic role of miR-378 in MDS. Interestingly, MDS cells do not form solid tumors in human body. However, in this study, SKM-1 cells were subcutaneously injected and solid tumors were formed in NOD/SCID mice. The same phenomenon was observed in another study (28), in which both SKM-1 cells and K562 cells were transplanted subcutaneously and solid tumors formed in immunodeficient mice. This discrepancy may attribute to the fact that the microenvironments provided by these mice are not identical to those for humans, although they are suitable for MDS cell-derived tumor growth. On the other hand, NOD/SICD mice lack both functional T- and B-cells (36), which may result in easier formation of MDS cell-derived solid tumors in these mice.

As described before, Wang et al (29) reported similar suppressive effect of miR-378 on colorectal cancer cells. However, the study of Wang and colleagues only clarified the cell cycle-related mechanisms involved in the action of miR-378, but did not further address the mechanisms underlying the miR-378-induced apoptosis. To explore the mechanisms underlying apoptosis caused by miR-378 in SKM-1 cells, we employed two bioinformatics algorithms to predict potetial taget genes of miR-378. Both bioinformatic analyses implied that anti-apoptotic protein gene Bcl-w is a putative target for miR-378. As a member of B cell lymphoma 2 (Bcl-2) family, Bcl-w plays an anti-apoptotic function in both healthy cells and cancer cells (37,38). Bcl-2 family of proteins, which are a group of important regulators in the mitochondriarelated apoptosis pathway, contain anti-apoptotic proteins (Bcl-2, Bcl-xl, Bcl-w) and pro-apoptotic proteins (Bax, Bad, Bid, Bak) (39). Bcl-w could inhibit mitochondria-mediated apoptosis by directly interacting with pro-apoptotic proteins Bax and Bad $(38,40)$. In addition to blocking apoptosis of mitochondrial pathway, it also protects cells from apoptosis by suppressing the activation of stress-activated protein kinase (41). Moreover, this anti-apoptotic protein has been found commonly expressed in various cancers (41-43), promoted tumor cell growth and invasion $(44,45)$. In this study, we proved that Bcl-w was a direct target of miR-378 using a dual-luciferase reporter assay. In agreement with this, western blotting showed that miR-378 reduced the expression of Bcl-w protein. By contrast, the Bcl-w mRNA level was not affected by ectopic expression of miR-378. All these findings indicated that miR-378 negatively regulated the expression of Bcl-w protein by directly binding the 3'UTR of Bcl-w mRNA.

In addition, we evaluated the expression of cleavedcaspase- 3 , cleaved-caspase- 8 and cleaved-caspase- 9 , which are critical regulators of apoptosis. There are two major pathways involved in apoptosis, i.e., the extrinsic death receptor pathway and the intrinsic mitochondrial pathway (46). The extrinsic pathway is triggered by the tumor necrosis factors and further leads to the cleavage and activation of caspase-8 (47). In the intrinsic mitochondrial pathway, caspase- 9 is activated by apoptosome, which is a complex that formed of cytochrome $c$ and apoptotic protease activating factor-1 (48). Both extrinsic pathway and intrinsic pathway lead to the cleavage and acti- vation of caspase-3 and finally cause apoptosis (49). In this study, we observed that enforced expression of miR-378 led to increased levels of cleaved caspase- $-3,-8$ and -9 , indicating that miR-378 induced apoptosis in SKM-1 cells via both extrinsic pathway and intrinsic pathway. Besides, we found that expression of pro-apoptotic protein Bax was notably upregulated in miR-378-overexpressing SKM-1 cells. Several studies have reported high ratio of Bcl-2 family pro-apoptotic proteins versus anti-apoptotic proteins correlates with increased apoptosis in MDS cells (50-52). A recent study proved that increased $\mathrm{Bax} / \mathrm{Bcl}-\mathrm{w}$ ratio contributed to the activation of caspase-dependent apoptosis (45). Based on these findings, we propose a potential mechanism behind apoptosis induced by overexpression of miR-378 in SKM-1 cells. The upregulation of Bax and downregulation of Bcl-w caused by miR-378 result in a remarkable increase in the $\mathrm{Bax} / \mathrm{Bcl}-\mathrm{w}$ ratio. Increased Bax/Bcl-w ratio directly or indirectly leads to activation of caspase-3, -8 and -9 that further induces caspase-dependent apoptosis. However, how miR-378 upregulates the expression level of Bax protein is still unknown.

In conclusion, this study pointed to a pronounced downregulation of miR-378 in MDS patients. Overexpression of miR-378 can suppress MDS/AML cell growth by promoting apoptosis and blocking G1/S-phase transition, at least, partly via negatively regulating Bcl-w and CDC40 expression. However, because each miRNA can regulate multiple target genes and the miRNA-regulated signaling pathways are complex, the specific mechanisms of miR-378 in the development of MDS are still needed to be elucidated in further studies. Recently, a study found that treatment of miRNA inhibitor could reverse the phenotypes of mouse model resembling MDS (13), suggesting that miRNA-based treatment may be a new strategy for therapy of MDS.

\section{Acknowledgements}

We are gratefultoChongqing Key Laboratory of Ophthalmology for the technical assistance. We also thank Mr. Nian Zhou and Miss Xinxin Li for their great help in this study. This study was supported by the National Natural Science Foundation of China (nos. 30971277 and 81250034), the Natural Science Foundation of Chongqing (CSTC, 2009BB5070), the bureau of Chongqing (2013-2-023), the Foundation of Chongqing Graduate Student Innovative Research (CYS14123) and the Project Foundation of Chongqing Municipal Education Committee (2013).

\section{References}

1. Adès L, Itzykson R and Fenaux P: Myelodysplastic syndromes. Lancet 383: 2239-2252, 2014.

2. Garcia-Manero G: Myelodysplastic syndromes: 2014 update on diagnosis, risk-stratification, and management. Am J Hematol 89: 97-108, 2014

3. Greenberg P, Cox C, LeBeau MM, Fenaux P, Morel P, Sanz G, Sanz M, Vallespi T, Hamblin T, Oscier D, et al: International scoring system for evaluating prognosis in myelodysplastic syndromes. Blood 89: 2079-2088, 1997.

4. Bejar R, Levine R and Ebert BL: Unraveling the molecular pathophysiology of myelodysplastic syndromes. J Clin Oncol 29: 504-515, 2011.

5. Bartel DP: MicroRNAs: Genomics, biogenesis, mechanism, and function. Cell 116: 281-297, 2004. 
6. Calin GA, Sevignani C, Dumitru CD, Hyslop T, Noch E, Yendamuri S, Shimizu M, Rattan S, Bullrich F, Negrini M, et al: Human microRNA genes are frequently located at fragile sites and genomic regions involved in cancers. Proc Natl Acad Sci USA 101: 2999-3004, 2004.

7. Alemdehy MF and Erkeland SJ: MicroRNAs: Key players of normal and malignant myelopoiesis. Curr Opin Hematol 19: 261-267, 2012.

8. Navarro A, Gaya A, Martinez A, Urbano-Ispizua A, Pons A, Balagué O, Gel B, Abrisqueta P, Lopez-Guillermo A, Artells R, et al: MicroRNA expression profiling in classic Hodgkin lymphoma. Blood 111: 2825-2832, 2008.

9. Rokah OH, Granot G, Ovcharenko A, Modai S, PasmanikChor M, Toren A, Shomron N and Shpilberg O: Downregulation of miR-31, miR-155, and miR-564 in chronic myeloid leukemia cells. PLoS One 7: e35501, 2012.

10. Wang J, Xiang G, Zhang K and Zhou Y: Expression signatures of intragenic miRNAs and their corresponding host genes in myeloid leukemia cells. Biotechnol Lett 34: 2007-2015, 2012.

11. Votavova H, Grmanova M, Dostalova Merkerova M, Belickova M, Vasikova A, Neuwirtova R and Cermak J: Differential expression of microRNAs in CD34 $4^{+}$cells of 5q- syndrome. J Hematol Oncol 4: $1,2011$.

12. Lee DW, Futami M, Carroll M, Feng Y, Wang Z, Fernandez M, Whichard Z, Chen Y, Kornblau S, Shpall EJ, et al: Loss of SHIP-1 protein expression in high-risk myelodysplastic syndromes is associated with miR-210 and miR-155. Oncogene 31: 4085-4094, 2012

13. Bhagat TD, Zhou L, Sokol L, Kessel R, Caceres G, Gundabolu K Tamari R, Gordon S, Mantzaris I, Jodlowski T, et al: miR-21 mediates hematopoietic suppression in MDS by activating TGF- $\beta$ signaling. Blood 121: 2875-2881, 2013.

14. Song SJ, Ito K, Ala U, Kats L, Webster K, Sun SM, JongenLavrencic M, Manova-Todorova K, Teruya-Feldstein J, Avigan DE, et al: The oncogenic microRNA miR-22 targets the TET2 tumor suppressor to promote hematopoietic stem cell selfrenewal and transformation. Cell Stem Cell 13: 87-101, 2013

15. Starczynowski DT, Kuchenbauer F, Argiropoulos B, Sung S Morin R, Muranyi A, Hirst M, Hogge D, Marra M, Wells RA, et al: Identification of miR-145 and miR-146a as mediators of the 5q- syndrome phenotype. Nat Med 16: 49-58, 2010.

16. Deng H, Guo Y, Song H, Xiao B, Sun W, Liu Z, Yu X, Xia T, Cui L and Guo J: MicroRNA-195 and microRNA-378 mediate tumor growth suppression by epigenetical regulation in gastric cancer. Gene 518: 351-359, 2013

17. Zhang GJ, Zhou H, Xiao HX, Li Y and Zhou T: MiR-378 is an independent prognostic factor and inhibits cell growth and invasion in colorectal cancer. BMC Cancer 14: 109, 2014

18. Sand M, Skrygan M, Georgas D, Sand D, Hahn SA, Gambichler T, Altmeyer P and Bechara FG: Microarray analysis of microRNA expression in cutaneous squamous cell carcinoma. J Dermatol Sci 68: 119-126, 2012

19. Yu BL, Peng XH, Zhao FP, Liu X, Lu J, Wang L, Li G, Chen HH and Li XP: MicroRNA-378 functions as an onco-miR in nasopharyngeal carcinoma by repressing TOB2 expression. Int J Oncol 44: 1215-1222, 2014

20. Yin JY, Deng ZQ, Liu FQ, Qian J, Lin J, Tang Q, Wen XM, Zhou JD, Zhang YY and Zhu XW: Association between mir-24 and mir-378 in formalin-fixed paraffin-embedded tissues of breast cancer. Int J Clin Exp Pathol 7: 4261-4267, 2014.

21. Chan JK, Kiet TK, Blansit K, Ramasubbaiah R, Hilton JF, Kapp DS and Matei D: MiR-378 as a biomarker for response to anti-angiogenic treatment in ovarian cancer. Gynecol Oncol 133: $568-574,2014$

22. Lee DY, Deng Z, Wang CH and Yang BB: MicroRNA-378 promotes cell survival, tumor growth, and angiogenesis by targeting $\mathrm{SuFu}$ and Fus-1 expression. Proc Natl Acad Sci USA 104: 20350-20355, 2007.

23. Pizzimenti S, Ferracin M, Sabbioni S, Toaldo C, Pettazzoni P, Dianzani MU, Negrini M and Barrera G: MicroRNA expression changes during human leukemic HL-60 cell differentiation induced by 4-hydroxynonenal, a product of lipid peroxidation. Free Radic Biol Med 46: 282-288, 2009.

24. Qian J, Lin J, Qian W, Ma JC, Qian SX, Li Y, Yang J, Li JY, Wang CZ, Chai HY, et al: Overexpression of miR-378 is frequent and may affect treatment outcomes in patients with acute myeloid leukemia. Leuk Res 37: 765-768, 2013.

25. Erdogan B, Facey C, Qualtieri J, Tedesco J, Rinker E, Isett RB Tobias J, Baldwin DA, Thompson JE, Carroll M, et al: Diagnostic microRNAs in myelodysplastic syndrome. Experimental hematology 39: 915-926 e912, 2011.
26. Nakagawa T, Matozaki S, Murayama T, Nishimura R, Tsutsumi M, Kawaguchi R, Yokoyama Y, Hikiji K, Isobe T and Chihara K: Establishment of a leukaemic cell line from a patient with acquisition of chromosomal abnormalities during disease progression in myelodysplastic syndrome. Br J Haematol 85: 469-476, 1993 .

27. Tiscornia G, Singer O and Verma IM: Production and purification of lentiviral vectors. Nat Protoc 1: 241-245, 2006

28. Yang B, Wang L, Luo X, Chen L, Yang Z and Liu L: SPAG6 silencing inhibits the growth of the malignant myeloid cell lines SKM-1 and K562 via activating p53 and caspase activationdependent apoptosis. Int J Oncol 46: 649-656, 2015.

29. Wang KY, Ma J, Zhang FX, Yu MJ, Xue JS and Zhao JS: MicroRNA-378 inhibits cell growth and enhances L-OHPinduced apoptosis in human colorectal cancer. IUBMB Life 66 645-654, 2014

30. Chen LT, Xu SD, Xu H, Zhang JF, Ning JF and Wang SF MicroRNA-378 is associated with non-small cell lung cancer brain metastasis by promoting cell migration, invasion and tumor angiogenesis. Med Oncol 29: 1673-1680, 2012.

31. Drexler HG, Dirks WG and Macleod RA: Many are called MDS cell lines: One is chosen. Leuk Res 33: 1011-1016, 2009.

32. Kaplan Y and Kupiec M: A role for the yeast cell cycle/splicing factor Cdc40 in the G1/S transition. Curr Genet 51: 123-140, 2007.

33. Hanahan D and Weinberg RA: The hallmarks of cancer. Cell 100: $57-70,2000$

34. Imada K: Immunodeficient mouse models of lymphoid tumors. Int J Hematol 77: 336-341, 2003.

35. Flavell DJ: Modelling human leukemia and lymphoma in severe combined immunodeficient (SCID) mice: Practical applications. Hematol Oncol 14: 67-82, 1996.

36. Shultz LD, Schweitzer PA, Christianson SW, Gott B, Schweitzer IB, Tennent B, McKenna S, Mobraaten L, Rajan TV, Greiner DL, et al: Multiple defects in innate and adaptive immunologic function in NOD/LtSz-scid mice. J Immunol 154: 180-191, 1995.

37. Gibson L, Holmgreen SP, Huang DC, Bernard O, Copeland NG, Jenkins NA, Sutherland GR, Baker E, Adams JM and Cory S: bcl-w, a novel member of the bcl-2 family, promotes cell survival. Oncogene 13: 665-675, 1996.

38. Yan W, Samson M, Jégou B and Toppari J: Bcl-w forms complexes with Bax and Bak, and elevated ratios of Bax/ $\mathrm{Bcl}-\mathrm{w}$ and $\mathrm{Bak} / \mathrm{Bcl}-\mathrm{w}$ correspond to spermatogonial and spermatocyte apoptosis in the testis. Mol Endocrinol 14: 682-699, 2000.

39. Adams JM and Cory S: The Bcl-2 protein family: Arbiters of cell survival. Science 281: 1322-1326, 1998.

40. Rodust PM, Fecker LF, Stockfleth E and Eberle J: Activation of mitochondrial apoptosis pathways in cutaneous squamous cell carcinoma cells by diclofenac/hyaluronic acid is related to upregulation of Bad as well as downregulation of Mcl-1 and Bcl-w. Exp Dermatol 21: 520-525, 2012

41. Lee HW, Lee SS, Lee SJ and Um HD: Bcl-w is expressed in a majority of infiltrative gastric adenocarcinomas and suppresses the cancer cell death by blocking stress-activated protein kinase/c-Jun NH2-terminal kinase activation. Cancer Res 63: $1093-1100,2003$

42. Wilson JW, Nostro MC, Balzi M, Faraoni P, Cianchi F, Becciolini A and Potten CS: Bcl-w expression in colorectal adenocarcinoma. Br J Cancer 82: 178-185, 2000.

43. Sanjmyatav J, Steiner T, Wunderlich H, Diegmann J, Gajda M and Junker K: A specific gene expression signature characterizes metastatic potential in clear cell renal cell carcinoma. J Urol 186: 289-294, 2011.

44. Bae IH, Park MJ, Yoon SH, Kang SW, Lee SS, Choi KM and Um HD: Bcl-w promotes gastric cancer cell invasion by inducing matrix metalloproteinase-2 expression via phosphoinositide 3-kinase, Akt, and Sp1. Cancer Res 66: 4991-4995, 2006.

45. Wang F, Liu M, Li X and Tang H: MiR-214 reduces cell survival and enhances cisplatin-induced cytotoxicity via down-regulation of Bcl212 in cervical cancer cells. FEBS Lett 587: 488-495, 2013.

46. Hengartner MO: The biochemistry of apoptosis. Nature 407 770-776, 2000

47. Wallach D, Varfolomeev EE, Malinin NL, Goltsev YV, Kovalenko AV and Boldin MP: Tumor necrosis factor receptor and Fas signaling mechanisms. Annu Rev Immunol 17: 331-367, 1999. 
48. Li P, Nijhawan D, Budihardjo I, Srinivasula SM, Ahmad M, Alnemri ES and Wang X: Cytochrome $c$ and dATP-dependent formation of Apaf-1/caspase-9 complex initiates an apoptotic protease cascade. Cell 91: 479-489, 1997.

49. Goldar S, Khaniani MS, Derakhshan SM and Baradaran B: Molecular mechanisms of apoptosis and roles in cancer development and treatment. Asian Pac J Cancer Prev 16: 2129-2144, 2015.

50. Boudard D, Vasselon C, Berthéas MF, Jaubert J, Mounier C, Reynaud J, Viallet A, Chautard S, Guyotat D and Campos L: Expression and prognostic significance of $\mathrm{Bcl}-2$ family proteins in myelodysplastic syndromes. Am J Hematol 70: 115-125, 2002 .
51. Parker JE, Fishlock KL, Mijovic A, Czepulkowski B, Pagliuca A and Mufti GJ: 'Low-risk' myelodysplastic syndrome is associated with excessive apoptosis and an increased ratio of pro- versus anti-apoptotic bcl-2-related proteins. Br J Haematol 103: 1075-1082, 1998 .

52. Parker JE, Mufti GJ, Rasool F, Mijovic A, Devereux S and Pagliuca A: The role of apoptosis, proliferation, and the Bcl-2related proteins in the myelodysplastic syndromes and acute myeloid leukemia secondary to MDS. Blood 96: 3932-3938, 2000 . 